\title{
Coupled map models for chaos in extended systems
}

\author{
M. S. Bourzutschky and M. C. Cross \\ Division of Physics, Mathematics and Astronomy, California Institute of Technology, Pasadena, \\ California 91125
}

(Received 21 June 1991; accepted for publication 14 November 1991)

\begin{abstract}
Coupled maps with conserved quantities are introduced as models for chaos in extended systems. The long-wavelength limit of a simple one-dimensional example is investigated in detail. A Langevin equation for this system is derived, and the applicability of the fluctuation-dissipation theorem is discussed. Several numerical experiments to investigate possible thermodynamic properties are described.
\end{abstract}

\section{INTRODUCTION}

In this paper we investigate possible reduced descriptions of extended dissipative chaotic systems, which are designed to describe the physics relevant on length scales large compared to the scale at which chaos can first be identified. For example, in Rayleigh-Bénard convection experiments at modest to large Rayleigh numbers, ${ }^{1}$ chaos occurs in the motion of a large number of convection rolls. Rather than attempt to solve the equations of motion starting with the full Navier-Stokes equation to obtain the detailed structure of each roll, we would consider something like a single roll as the smallest length scale. Our reduced description should then describe the collective "hydrody. namics" of many such rolls, analogous to the reduced description that thermodynamics and hydrodynamics provide for equilibrium systems. Another example is the disorder observed in surface waves at large driving amplitudes, ${ }^{2}$ where the basic element might be a small domain of ripples.

An obvious, but perhaps not fully appreciated, remark is that the new feature of dissipative systems is not the presence of chaos, which is presumably also responsible for the ergodic behavior in thermodynamics, but the absence of a Hamiltonian which would provide a simple measure for averaging over the phase space. The constant energy surface with uniform weighting in the measure of the microcanonical formulation of Hamiltonian systems is perhaps replaced by a strange attractor of some a priori unknown measure. The question then arises of how much of the simplification yielded by the reduced description of thermodynamics and hydrodynamics persists in dissipative systems.

\section{COUPLED MAPS WITH CONSERVATION LAWS}

Much of our knowledge about chaotic dynamical systems with a small number of degrees of freedom has been gained from the study of discrete iterated maps. ${ }^{3}$ Constructing a lattice of single maps, with a prescribed coupling between sites, provides a convenient method for simulating extended systems with highly nonlinear local interactions. Such coupled map lattices have indeed been used to investigate pattern formation and spatially extended chaos in a variety of contexts. ${ }^{4}$ Here, we investigate maps whose (discrete) time dynamics satisfies a simple conservation law, similar to the continuity equation expressing mass conservation in fluid flow. There are a number of reasons for imposing conservation laws.

First, conservation laws occur naturally in many situations we might try to describe. For example, in RayleighBénard convection the average density is conserved. In surface wave experiments, the average level of fluid in the container is conserved. However, we emphasize that conservation laws are by no means inevitable, since our model equations are no longer the full equations of motion. Furthermore, external couplings can destroy conservation laws one would otherwise expect. For example, in a RayleighBénard convection cell, the average horizontal momentum is no longer conserved due to coupling to the top and bottom plates of the container.

Second, apparent conservation laws can arise through broken symmetries. An example is the superfluid velocity in a Bose condensed system $\mathbf{v}_{s} \propto \nabla \phi$ with $\phi$ the phase of the macroscopic wave function: The dynamics of $\mathrm{v}_{s}$ is governed by a conservation law, in fact deriving from the equation for $\phi$ that describes the broken gauge symmetry:

$$
\partial_{t} \phi=F\{\nabla \phi, \ldots\}
$$

so that

$$
\partial_{i} \mathbf{v}_{s}=\nabla F\left\{\mathbf{v}_{s}, \ldots\right\},
$$

which has the form of a conservation law. Here, $F$ cannot depend on $\phi$ itself by the basic phase symmetry. An analogous case in nonequilibrium systems is the KuramotoSivashinsky equation: ${ }^{5}$

$$
\partial_{t} u(x, t)=-\partial_{x}^{2} u-\partial_{x}^{4} u-u \partial_{x} u .
$$

This equation may be derived as the dynamics of a phase variable $\phi$ which here characterizes displacements of a periodic pattern (broken translational symmetry) with $u$ $=\partial_{x} \phi$. A reduced description of the KuramotoSivashinsky equation in terms of a Burgers equation with stochastic noise has been given in Ref. 6 .

Finally, it is known that in equilibrium systems interesting long-length scale dynamics usually derives from conservation laws: In the absence of these, long wavelength properties often reduce to simple averages over independent, rapidly fluctuating microscopic regions, usually yielding uninteresting results. We expect the same to be true for dissipative systems, although there may still be 
interesting questions on how to characterize the shortlength scale behavior in systems without conserved quantities.

With these considerations, we consider the following simple one-dimensional coupled map model which satisfies a continuity equation:

$$
y_{i}^{n+1}=y_{i}^{n}+g\left(y_{i+1}^{n}, y_{i}^{n}\right)-g\left(y_{i}^{n}, y_{i-1}^{n}\right),
$$

where $y$ is a continuous scalar variable, $n$ is a discrete "time" index, and $i$ is a lattice index running from 1 to the system size $L$. The role of the current term in the continuity equation is played by the scalar function $g$, which satisfies $g(x, y)=-g(y, x)$ to provide parity symmetry $i$ $\rightarrow-i$. We enforce periodic boundary conditions:

$$
y_{i+L}^{n}=y_{i}^{n} \text {. }
$$

It is evident that there is a locally conserved "mass" $Q$ independent of $n$ :

$$
Q=\sum_{i=1}^{L} y_{i}^{n}
$$

Two different choices for $g$ are considered here and will be referred to as "model A" and "model B." Model A is defined by

$$
g(x, y)=f(x)-f(y),
$$

where

$$
f(x)=a x+b z(1-z)
$$

with

$$
z \equiv|x|(\bmod 1),
$$

where $a$ and $b$ are positive constants. Model B is defined by

$$
g(x, y)=2 f(x-y) /\left(1+x^{2}+y^{2}\right),
$$

with

$$
f(x)=c x+a \sin (b x),
$$

where $a, b$, and $c$ are constants. Most results quoted in this paper were obtained with model A.

\section{CHARACTERIZATION OF SPATIALLY EXTENDED CHAOS}

We now describe some simple numerical experiments which shed some light on the nature of the extended chaotic state and which motivate the search for a simple long wavelength description.

For $b=0$, model A evidently reduces to a finite difference approximation to the one-dimensional diffusion equation. Almost all initial configurations decay to a spatially uniform state $y_{i}^{n}=$ const. At intermediate $b$, one observes local chaotic fluctuations on ordered spatial patterns, as seen in many coupled maps without conservation laws. ${ }^{4}$ Here, we focus on $b$ large enough such that all spatial order is destroyed. For a map with $L$ lattice sites, we can compute a set of $L$ Lyapunov exponents which quantify the exponential divergence of trajectories due to the chaotic time evolution. ${ }^{7}$ In Fig. 1 we show the Lyapunov

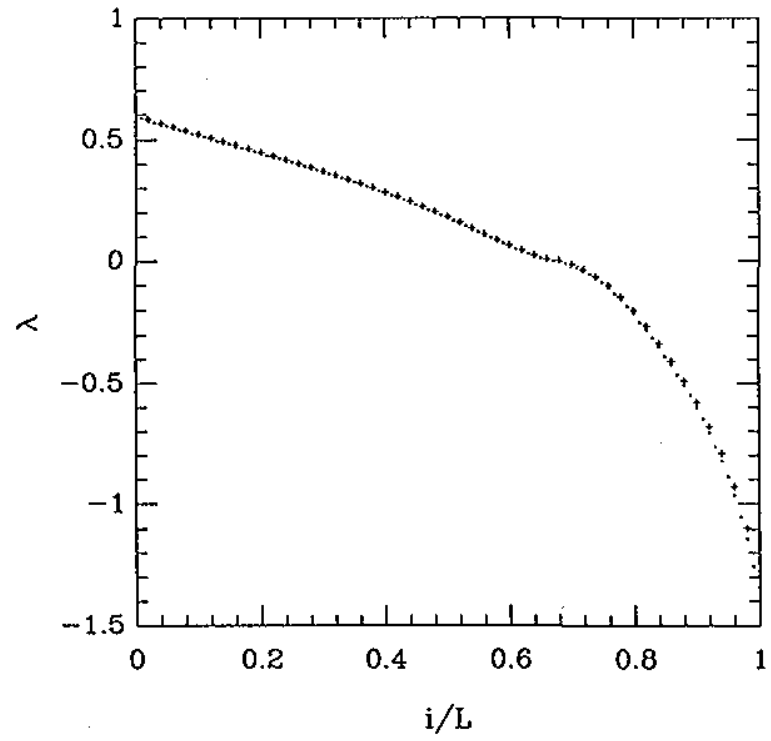

FIG. 1. Lyapunov exponents for model A with $a=0.4, b=1.3$, for $L$ $=50$ (pluses) and $L=100$ (points), averaged over 100000 iterations.

spectrum for two different lattice sizes, clearly showing positive exponents. There are several additional features of the Lyapunov spectrum worth pointing out. For example, the Lyapunov (or Kaplan-Yorke ${ }^{8}$ ) dimension cannot be defined here because the sum of the Lyapunov exponents $\lambda$ is greater than zero, which is possible because of the discrete dynamics: For partial differential equations with continuous space and time symmetry describing dissipative systems, this sum would always be negative through the appearance of negative $\lambda$ of arbitrarily large magnitude at the low end of the spectrum. ${ }^{9}$

Another interesting feature of the Lyapunov spectrum (cf. Fig. 1) is an approximate point of inflection near $\lambda$ $=0$. Obviously, one exponent is always trivially zero because of the conservation law (the vector perpendicular to the hyperplane defined by $\Sigma_{i=1}^{L} y_{i}^{n}=$ const does not change its length under the dynamics), but perhaps a connection can be established between small but nonzero exponents and the slow modes of the system. A point of inflection also appears in the spectrum of model $\mathrm{B}$, indicating that it might be quite generic. Some mathematical arguments for singularities in the density of Lyapunov exponents exist for certain models of intermittent turbulence. ${ }^{10}$

The intensive density of Lyapunov exponents suggested by Fig. 1 is consistent with a lattice that has short range correlations. We see this more directly by measuring the equal time correlation function $S(r)$ (cf. Fig. 2) defined as

$$
S(r)=\left\langle\left(y_{i}^{n}-\langle y\rangle\right)\left(y_{i+r}^{n}-\langle y\rangle\right)\right\rangle,
$$

where $\langle\ldots\rangle$ denotes averaging over both $i$ and $n$. While $S(r)$ does not have a simple exponential form, it decays rapidly, indicating a finite correlation length of approximately two lattice spacings. Appealing to the central limit theorem, we now expect that fluctuations of spatially averaged quantities will have a Gaussian distribution, since 




FIG. 2. Spatial correlation function $S(r)$ for model A with $L=512, a$ $=0.4, b=1.28$, averaged over four million iterations.

the average will be over many uncorrelated regions. This will be true even if the decay of correlations is algebraic rather than exponential, as long as the relevant power is large enough. As a simple example, we consider the spatial Fourier transform of the lattice amplitudes:

$$
y_{k}^{n}=\frac{1}{\sqrt{L}} \sum_{j=1}^{L} e^{2 \pi i j k} y_{j}^{n}
$$

where $k$ ranges from $0-0.5$ in discrete steps of the reciprocal lattice vector $1 / L$. The probability distribution $D\left(\Delta y_{k}\right)$ of fluctuations in the Fourier amplitudes $\Delta y_{k}^{n}=y_{k}^{n}-\left\langle y_{k}^{n}\right\rangle$ for fixed $k$, and averaged over $n$, is shown in Fig. 3(a), together with a Gaussian fit, which evidently describes the distribution well. Here, $D\left(\Delta y_{k}\right)$ should be contrasted with the distribution $D\left(\Delta y_{i}\right)$ of fluctuations of a single lattice amplitude, shown in Fig. 3(b). The latter distribution is, of course, strongly affected by the choice of map function, and cannot be expected to have a simple form. Also note that the width of $D\left(\Delta y_{k}\right)$ scales with wave vector, which is related to the vanishing of the structure factor $S(k)$ as $k \rightarrow 0$ in this model, which we will comment on further below.

\section{STRUCTURE FACTOR AND LANGEVIN EQUATION}

To investigate the long-wavelength, low-frequency behavior of the continuum limit of the map, we now derive a Langevin equation ${ }^{11}$ meant to capture the important long wavelength features of the model.

In Fourier space, the linearized Langevin equation is effectively an expansion of the equation of motion in powers of the wave vector $k$. The conservation law and the parity symmetry evidently require the lowest order term to be $\sim k^{2}$. We then assume that the effect of all short wavelength corrections to the equation of motion can be combined into a stochastic noise term. With these assumptions,
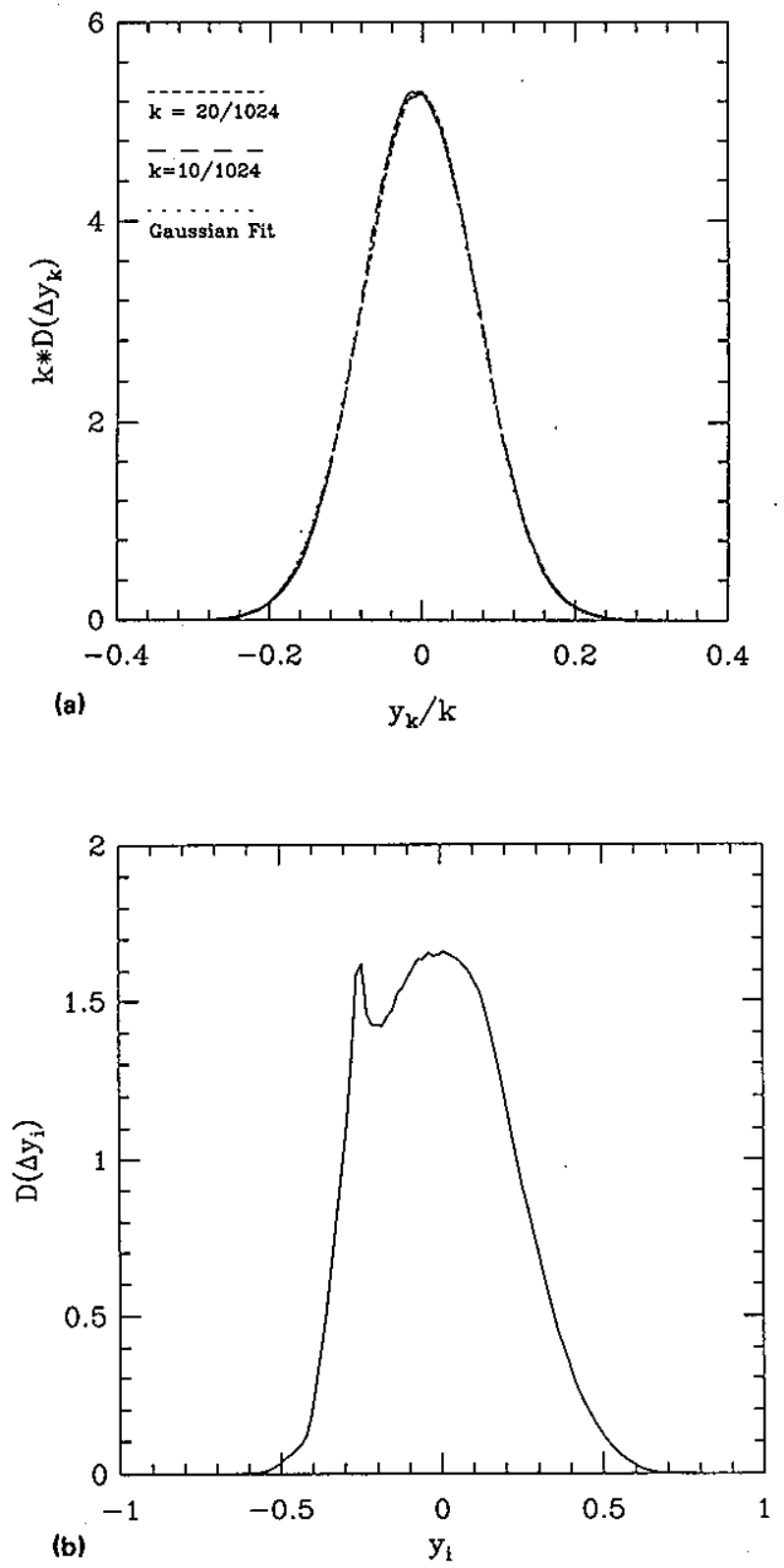

FIG. 3. Probability distributions of fluctuations for model A with $L$ $=1024, a=0.4$, and $b=1.3$, averaged over two million iterations. (a) Distribution $D\left(\Delta y_{k}\right)$ of Fourier amplitudes for wave vectors $k \Rightarrow 20$ / 1024 and $k=10 / 1024$. (b) Distribution $D\left(\Delta y_{i}\right)$ of a single lattice amplitude.

we therefore hypothesize the following long wavelength equation for model $\mathrm{A}$ :

$$
\partial y=D \partial_{x}^{2} y+\partial_{x}^{2} \eta(x, t)
$$

The deterministic part is identical to the linear diffusion equation with diffusion constant $D$. The noise $\eta$ is written as a second-order derivative, because it is generated by the map function $f$ in Eq. (6), which effectively appears under two spatial derivatives. We will assume that $\eta$ is Gaussian and delta-correlated, with zero mean and second moment

$$
\left\langle\eta(x, t) \eta\left(x^{\prime}, t^{\prime}\right)\right\rangle=A \delta\left(x-x^{\prime}\right) \delta\left(t-t^{\prime}\right) .
$$

Nonlinear terms may be added to Eq. (10). However, naively, one would expect their effect to be small at long 
wavelengths, since the noise averages to small values there. In recent years, Langevin models similar to Eq. (10) have been studied intensively because of the possibility that nonlinear terms can, in fact, lead to nonintegral powers of $k$ in the correlation function and long-time tails in the dynamics through the buildup of the noise at long wavelengths. ${ }^{12}$ The treatment of such singular equations is typically done using renormalization group transformations. ${ }^{13}$ In the present case, simple dimensional analysis (power counting) shows that no relevant nonlinear terms are likely to appear in Eq. (10). As an example, we will consider the case where an additional term $\lambda \partial_{x}^{2} y^{2}$, which satisfies the conservation law and parity symmetry, appears in the equation of motion. To study the effect at long wavelengths, we perform a scaling transformation $x \rightarrow b x, t \rightarrow b^{z} t$, and $y \rightarrow b^{\chi} y$, where $z$ and $\chi$ are scaling exponents to be determined. Including the nonlinear term, Eq. (10) then becomes

$$
\partial_{y} y=D b^{z-2} \partial_{x}^{2} y+\lambda b^{\chi+z-2} \partial_{x}^{2} y^{2}+b^{z / 2-\chi-5 / 2} \partial_{x}^{2} \eta .
$$

For $\lambda=0$, scaling is evidently achieved for $z=2$ and $\chi=$ $-3 / 2$. A nonzero $\lambda$ gets rescaled by $b^{\chi+z-2}=b^{-3 / 2}$. As $b$ is increased (i.e., the length scale considered gets larger), the rescaled $\lambda$ decreases, therefore becoming irrelevant in the renormalization group sense. It is easy to check that all analytic nonlinear terms consistent with the symmetries one might add to Eq. (10) are similarly irrelevant. Relevant nonlinearities will therefore only be expected to appear if the conservation law or the parity symmetry is broken. An example for the latter has been considered in a model of sand flowing down an incline, ${ }^{14}$ where a term of the form $\lambda \partial_{x} y^{2}$ has been included.

Given Eq. (10), the long-wavelength form of the dynamic structure factor $S(k, \omega)$ can now easily be calculated:

$$
S(k, \omega)=A D k^{4} /\left[\left(D k^{2}\right)^{2}+\omega^{2}\right] .
$$

As shown in Fig. 4(a) and (b), we find numerically results consistent with

$$
\begin{aligned}
& S(k, t)=S(k) e^{-D k^{2} t}, \\
& S(k)=A k^{2},
\end{aligned}
$$

which, upon Fourier transforming in time, is seen to be consistent with Eq. (12).

The vanishing of the static structure factor $S(k)$ as $k \rightarrow 0$ for model A is related to the extra symmetry in the noise, which enters through the current $g$ under effectively two spatial derivatives in Eq. (6). This symmetry is broken in model $\mathrm{B}$, and a numerical computation of $S(k)$ for model B (cf. Fig. 5) indeed shows that $S(k) \rightarrow$ const as $k \rightarrow 0$.

\section{LINEAR RESPONSE AND FLUCTUATION-DISSIPATION THEOREM}

An interesting suggestion has recently been made ${ }^{15}$ that a useful "temperature" might be defined for nonequilibrium systems via the fluctuation-dissipation theorem.

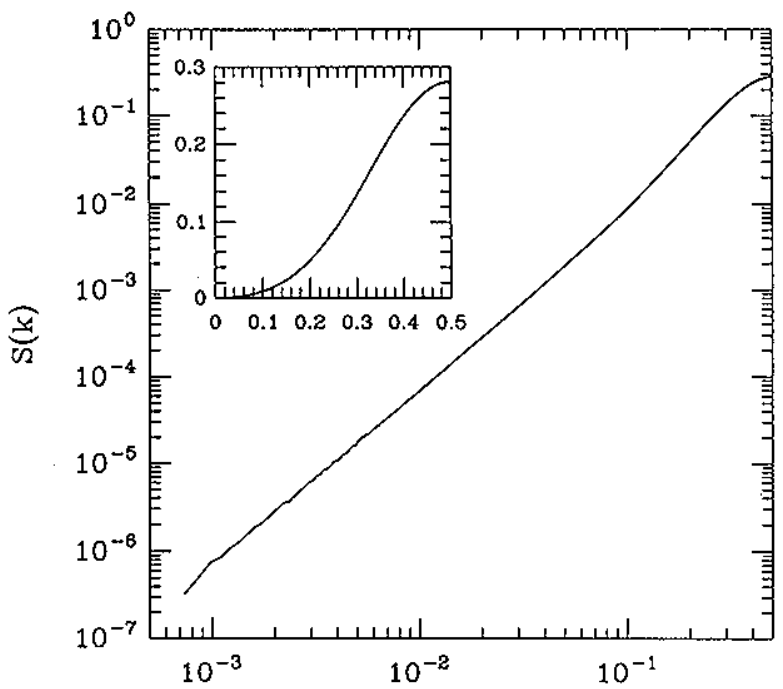

(a)

k

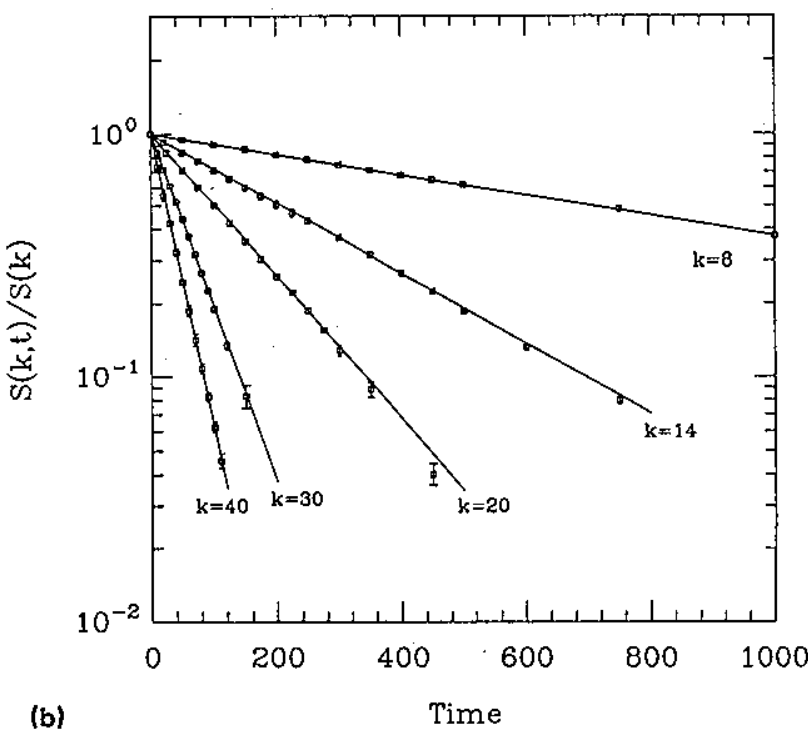

FIG. 4. Structure factors for model A with $a=0.4$ and $b=2.0$. (a) Static structure factor $S(k), L=8192$. (b) Dynamic structure factor $S(k, t)$ normalized by $S(k)$, for $L=1024$. The wave vector $k$ is given in units of $1 / L$.

For near equilibrium thermodynamic systems, this theorem provides a relation between the temperature $T$, the dynamic structure factor $S(k, \omega)$, and the susceptibility $\chi(k, \omega)$ :

$$
T=\omega S(k, \omega) / 2 \operatorname{Im}(\chi(k, \omega)) .
$$

In near equilibrium systems, this relation is true for all $k$ and $\omega$. Using appropriate dispersion relations, ${ }^{16}$ we can effectively integrate Eq. (15) over $\omega$ to relate $T$ to the static structure factor and static response functions:

$$
T=S(k, t=0) / \chi(k, \omega=0) .
$$

Following Ref. 15, we now investigate the question whether the right-hand side of Eq. (15), perhaps in the limit $k \rightarrow 0$ and $\omega \rightarrow 0$, can be considered as the definition for 


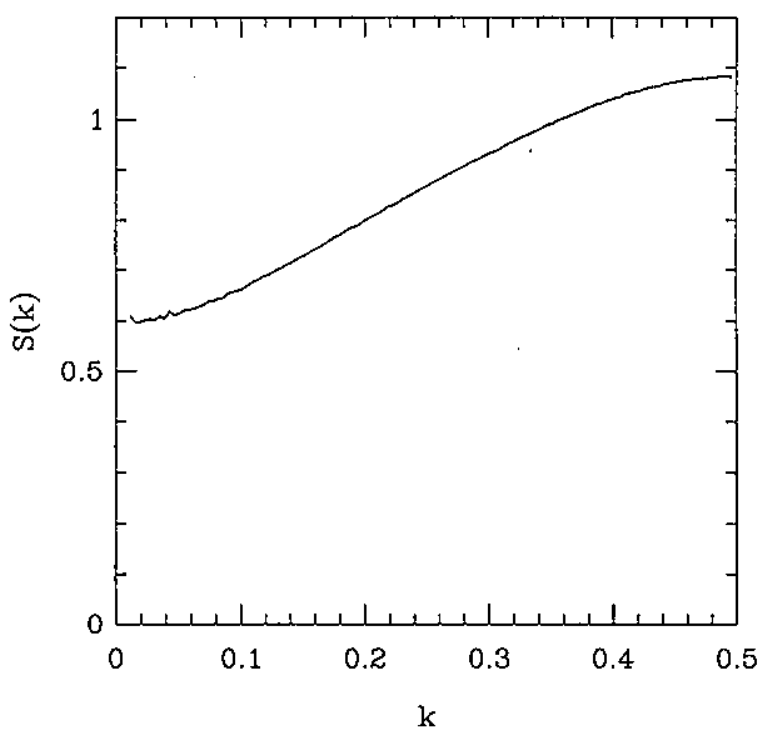

FIG. 5. Structure factor for model $B$ with $L=256, a=0.2, b=3.1416$, and $c=-0.6$, averaged over two million iterations.

an effective "temperature" $T_{e}$ having useful properties even for nonequilibrium systems. For example, $T_{e}$ might provide constraints on the effective noise term in the Langevin equation. Another useful application for $T_{e}$ would be the analogy of a thermometer: If two coupled systems have the same value of $T_{e}$, they should be in equilibrium, i.e., there should be no net flow of $Q$ between them.

Accordingly, following Ref. 15, we study the response of a coupled map lattice to a small applied field, chosen so as to preserve the conservation law and reflection symmetry of the map. The equation of motion Eq. (3) is thus modified to

$$
\begin{aligned}
y_{i}^{n+1}= & y_{i}^{n}+g\left(y_{i+1}^{n}, y_{i}^{n}\right)-g\left(y_{i}^{n}, y_{i-1}^{n}\right)+h_{i+1}^{n} \\
& -2 h_{i}^{n}+h_{i-1}^{n},
\end{aligned}
$$

where we concentrate on static fields $h_{j}^{n}=h \cos (2 \pi j k)$ with fixed wave number $k$. The static susceptibility $\chi_{k}$ is then

$$
\chi_{k}=\lim _{h \rightarrow 0}\left(2\left\langle y_{k}^{n}\right\rangle / h\right)
$$

where averaging is over $n$. Numerical calculations of $\chi_{k}$ for models A and B are shown in Fig. 6(a) and (b), respectively. At long wavelengths, we find $\chi_{k}$ to be essentially independent of $k$. This result can easily be understood in terms of the Langevin equation by including an additional term $\partial_{x}^{2} h$ on the right-hand side of Eq. (10). Since the applied field $h$ should be uncorrelated with the effective internal noise $\eta$, one immediately finds that $\chi(k)=1 / D$. We have checked the consistency of this result by comparing the values of $D$ obtained from $\chi(k)$ and $S(k, \omega)$ for model A [cf. Figs. 4(b) and 6(a)]. We find numerically that $D=0.45 \pm 0.02$ from $\chi(k)$ and $D=0.470 \pm 0.008$ from $S(k, \omega)$, showing good agreement

Returning to the fluctuation-dissipation ratio Eq. (16), we now deduce for model $\mathrm{A}$ that $T_{e} \sim k^{2}$. In the long-
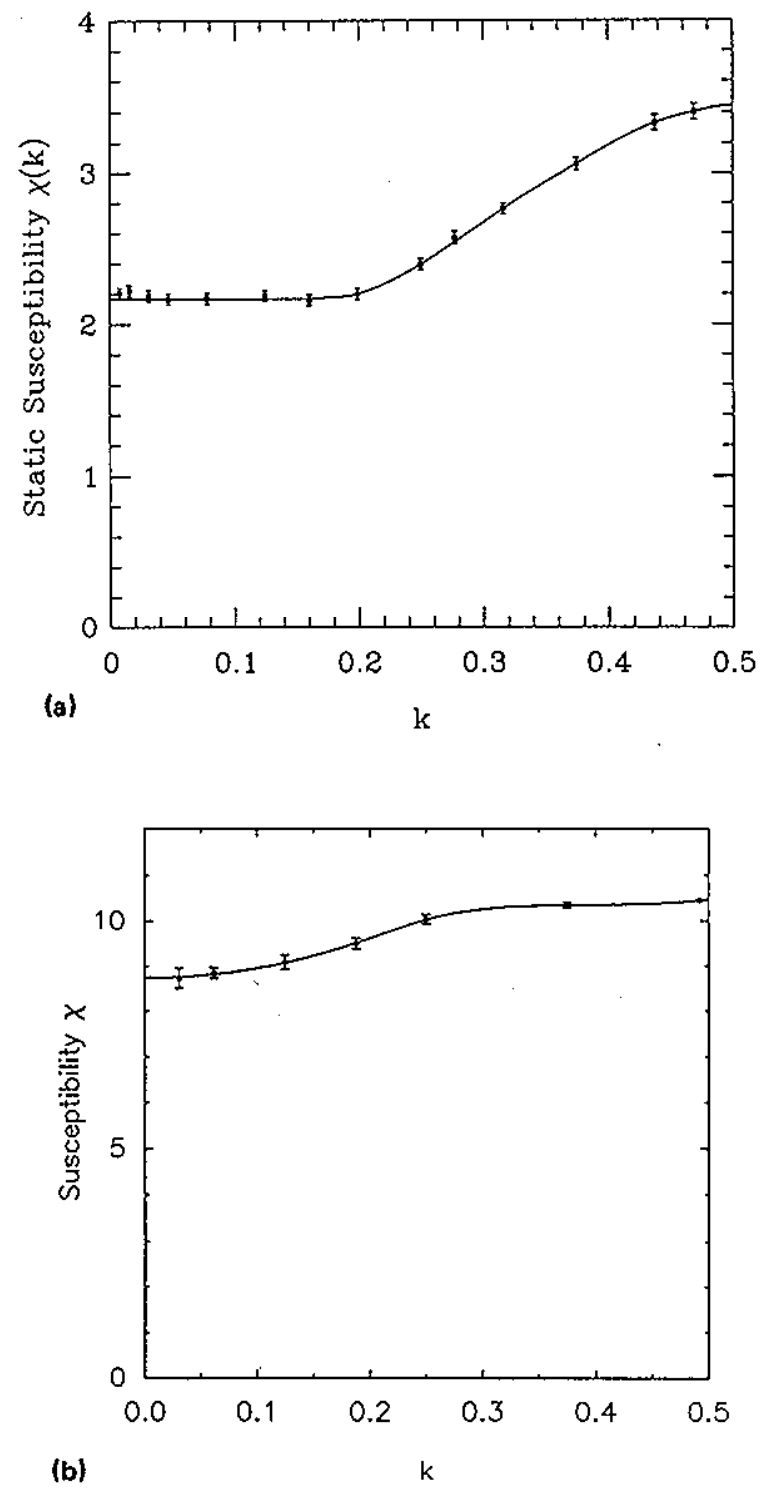

FIG. 6. Static susceptibility $\chi_{k}$. (a) Model A with $a=0.4, b=2.0$. (b) Model B with $a=0.2, b=3.1416$, and $c=-0.6$.

wavelength limit, this effective temperature always vanishes, indicating that $T_{e}$ does not have the desired properties. At the simplest level, the $k^{2}$ dependence of the effective temperature in model $\mathbf{A}$ comes from the extra gradient in the noise term of Eq. (10) as compared with the conventional ansatz for diffusional Langevin equations. However, the breakdown of the constant ratio between response and correlations can be traced to a more general flaw in the formulation, namely, our lack of knowledge of how the applied field couples to the probability functional of the system. For Hamiltonian systems, the applied fields are known to appear in the thermodynamic equations together with their conjugate fields; e.g., a magnetic field $\mathscr{H}$ appears in the internal energy through a relation of the form $\mathscr{H} d M$, where $M$ is the magnetization. For nonequilibrium systems, we do not have a corresponding formalism.

A simple example may be useful here to illustrate the problem. Consider the long wavelength description of the 
dynamics of a single conserved variable in an equilibrium system. The thermodynamics may be defined through the entropy $S$ and its density $s$ :

$$
\begin{aligned}
S= & S_{0}+\int\left\{s^{\prime} \delta q(x)+\frac{1}{2} K[\delta q(x)]^{2}\right. \\
& -h(x) \delta q(x)\} d^{d} x,
\end{aligned}
$$

where $s^{\prime}=\left.(\partial s / \partial q)\right|_{q=q_{0}}$, and $K=\left.\left(\partial^{2} s / \partial q^{2}\right)\right|_{q=q_{0}}$ (which plays the role of an elastic constant) and we have expanded in small fluctuations $\delta q=q-q_{0}$ about a uniform equilibrium value $q_{0}$. For conserving dynamics, one obtains from Eq. (19) the equation of motion: ${ }^{13}$

$$
\begin{aligned}
\dot{\delta} q & =\gamma \nabla^{2}\left(\frac{\delta S}{\delta q}\right)+\nabla \eta(x, t) \\
& =\gamma K \nabla^{2} \delta q+\nabla \eta(x, t)-\gamma \nabla^{2} h(x, t),
\end{aligned}
$$

where $\gamma$ is a kinetic coefficient and $\eta$ is a Gaussian stochastic noise term with strength $F$ :

$$
\left\langle\eta(x, t) \eta\left(x^{\prime}, t^{\prime}\right)\right\rangle=F \delta\left(x-x^{\prime}\right) \delta\left(t-t^{\prime}\right) .
$$

For an applied field $h(x, t)=h_{k \omega} e^{i k x-i \omega t}$ the average response $\delta q=\delta q_{k \omega} e^{i k x-i \omega t}$ calculated from Eq. (20) yields a susceptibility:

$$
\chi(k, \omega)=\delta q_{k \omega} / h_{k \omega}=\gamma k^{2} /\left(-i \omega+\gamma K k^{2}\right) .
$$

Similarly, one obtains the dynamic structure factor:

$$
S(k, \omega)=\left\langle\left(\delta q_{k \omega}\right)^{2}\right\rangle=k^{2} F /\left[\omega^{2}+\left(\gamma K k^{2}\right)^{2}\right],
$$

and, consequently, the static structure factor:

$$
S(k)=\left\langle\left(\delta q_{k}\right)^{2}\right\rangle=F / \gamma K .
$$

However, averaging over the probability distribution $e^{-F /}$ $T$ shows that

$$
\left\langle\left(\delta q_{k}\right)^{2}\right\rangle=2 T / K,
$$

so that the noise strength is related to the thermodynamic temperature via

$$
F=2 \gamma T \text {. }
$$

Using Eqs. (22), (23), and (26), both the static and dynamic fluctuation-dissipation theorem Eq. (15) are immediately verified in the long wavelength limit.

Let us now repeat this analysis in the way we are forced to for a nonequilibrium system. Instead of defining the coupling to an external field through Eq. (19), we need to introduce a field $\bar{h}$ into the dynamics; and we do not know the division of the diffusion constant $D$ into stiffness and kinetic parameters:

$$
\dot{\delta} q=D \nabla^{2} \delta_{q}+\nabla \eta(x, t)-\nabla^{2} \bar{h}(x, t) .
$$

From this we obtain

$$
\begin{aligned}
& \bar{\chi}(k, \omega)=\delta q_{k \omega} / \bar{h}_{k \omega}=k^{2} /\left(-i \omega+D k^{2}\right), \\
& S(k, \omega)=k^{2} F /\left[\omega^{2}+\left(D k^{2}\right)^{2}\right],
\end{aligned}
$$

so that, for the proposed definition of an effective temperature

$$
T_{e}=\omega S(k, \omega) / 2 \operatorname{Im}(\bar{\chi}(k, \omega)),
$$

we find $T_{e}=F / 2$.

Comparing with Eq. (26) for the equilibrium system we obtain

$$
T_{e}=\gamma T
$$

depending on the kinetic coefficient $\gamma$. Without further knowledge about $\gamma$ and its $k$ and $\omega$ dependence, $T_{e}$ cannot be expected to measure $T$. These are exactly the results found for our coupled map systems.

Similar conclusions apply to model B. Although in this case $S(k)$ and $\chi(k)$ both approach a constant as $k \rightarrow 0$, so that $S(k) / \chi(k) \rightarrow$ const, we would not expect this value to have the same importance as in equilibrium systems. It is also clear comparing Figs. 5 and $6(\mathrm{~b})$ that $S(k) / \chi(k)$ is' $k$ dependent for large $k$.

\section{TEMPERATURE BATHS}

In this section, we describe some numerical experiments that may provide alternative tests for the existence of an underlying thermodynamic principle in the behavior of coupled map lattices with conservation laws. In thermodynamic systems, important consequences follow from the existence of an additive local entropy function, which only depends on the (conserved) quantities, e.g., the energy. For example, maximizing the entropy determines how a given conserved total energy $E$ is divided up between two coupled systems in equilibrium and introduces the concept of the thermodynamic temperature. We now propose a "heat-bath algorithm" to test whether an analogous quantity to the entropy, which should only depend on the conserved quantity $Q$, exists in our coupled map models.

Consider, then, two coupled map lattices, with different equations of motion, e.g., different parameters $a$ and $b$ in Eq. (3). Let the conserved quantities in the two maps be $Q_{1}$ and $Q_{2}$. Now bring the two lattices into contact, by introducing a coupling term between them. The interaction will, in general, cause a current to flow between the two systems, until the conserved quantities reach new values $Q_{1}^{\prime}$ and $Q_{2}^{\prime}$, say, with the constraint $Q_{1}+Q_{2}=Q_{1}^{\prime}+Q_{2}^{\prime}$. If there exists an additive entropy, maximizing the total entropy will then uniquely determine $Q_{1}^{\prime}$ and $Q_{2}^{\prime}$.

The ideas in the preceding paragraph imply the transitivity of equilibrium: If $X$ is in equilibrium with $Y$, and $Y$ is in equilibrium with $Z$, then $X$ is in thermal equilibrium with $Z$. We test this transitivity by using three different coupled maps $X, Y$, and $Z$, obtained from model $\mathrm{A}$ by the choice of different parameters $a$ and $b$. Starting with a random initial configuration with total $Q=0, Y$ and $Z$ are, in turn, coupled to $X$ and iterated until steady state is achieved. [Coupling between two lattices is implemented by introducing an additional term $d\left(y^{1}-y^{2}\right)$ in the equation of motion of the endpoints of the two lattices, where $d$ is an adjustable coupling strength. The two lattices are arranged on a ring, so there are two such terms.] Steady state is characterized by new values of the conserved quan- 
tities $Q_{y}$ and $Q_{z}$ in $Y$ and $Z$. If the lattice sizes $L_{x}, L_{y}$, and $L_{z}$ are chosen such that $L_{y}=L_{z} \ll L_{x}$, then the change in the mean value of $Q_{x}$ should be small, so $X$ should act approximately as a heat bath with constant "temperature" throughout the coupling with $Y$ or $Z$. This implies that, when $Y$ and $Z$ are coupled after their "thermalization" with $X$, they should be in equilibrium, i.e., $Q_{y}$ and $Q_{z}$ should not change.

In Fig. 7(a)-(c) we illustrate the sequence of events described above by plotting the time evolution of $Q$ for three situations: The thermalization of two small lattices $Y$ and $Z$ with a large lattice $X$ is shown in Fig. 7 (a) and (b); in Fig. 7 (c) we test for equilibrium of $Y$ and $Z$ when initialized with the values of $Q$ generated from the equilibration with $X$. The transitivity of equilibrium appears to hold very well, with the tiny shifts in $Q_{y}$ and $Q_{z}$ actually seen qualitatively consistent with those expected due to the finite size of $X$.

Several tests can now be undertaken to check the consistency of the thermal equilibrium picture. To verify that each lattice reaches local equilibrium, we accumulate the time average of all lattice amplitudes separately, once $Q$ in each lattice no longer changes. In Fig. 8 we show the results for maps $X$ and $Z$. Except very close to the interface, the local means are indeed the same throughout each lattice, supporting the idea of local equilibrium. We also test whether the equilibrium values of $Q$ reached are independent of the coupling strength $d$. In Fig. 9, we show the equilibrium values of $Q_{y}$ and $Q_{z}$ as a function of $d$; no significant dependence is found. We have also checked various nonlinear coupling terms, like $d \sin \left(y^{1}-y^{2}\right)$. As long as the coupling term is odd under the interchange of $y^{1}$ and $y^{2}$, the equilibrium values of $Q$ obtained numerically are the same.

An alternative test for thermodynamic equilibrium consists of coupling three maps arranged on a ring, and monitoring the current flowing around the loop. If the three maps reach a consistent equilibrium, there should be no net current flow. In our numerical simulations of this situation, we do find persistent currents, but they are small compared to the shifts of the means between two lattices, as is evident in Fig. 10, where we show a typical time average of the local means. The persistent currents are barely discernable as a nonzero slope of the profile of the means across each lattice. Presently, our numerical results indicate that the magnitude of the persistent currents scales as $d / L$, where $L$ is the system size and $d$ is the coupling strength. A current scaling as $1 / L$ leads to a constant offset in $Q$, and would question the thermodynamic equilibrium picture. However, more numerical simulations on bigger systems with smaller coupling strengths are needed to resolve this issue.

Assuming that the thermodynamic picture does hold, we can test it further by checking whether the distribution of $Q$ between the three maps is consistent with that found in the temperature bath experiments where two maps are coupled at a time. To do this, the size $L_{x}$ of one of the three lattices is increased, and the corresponding rearrangement of $Q$ determined. The infinite system limit is approached
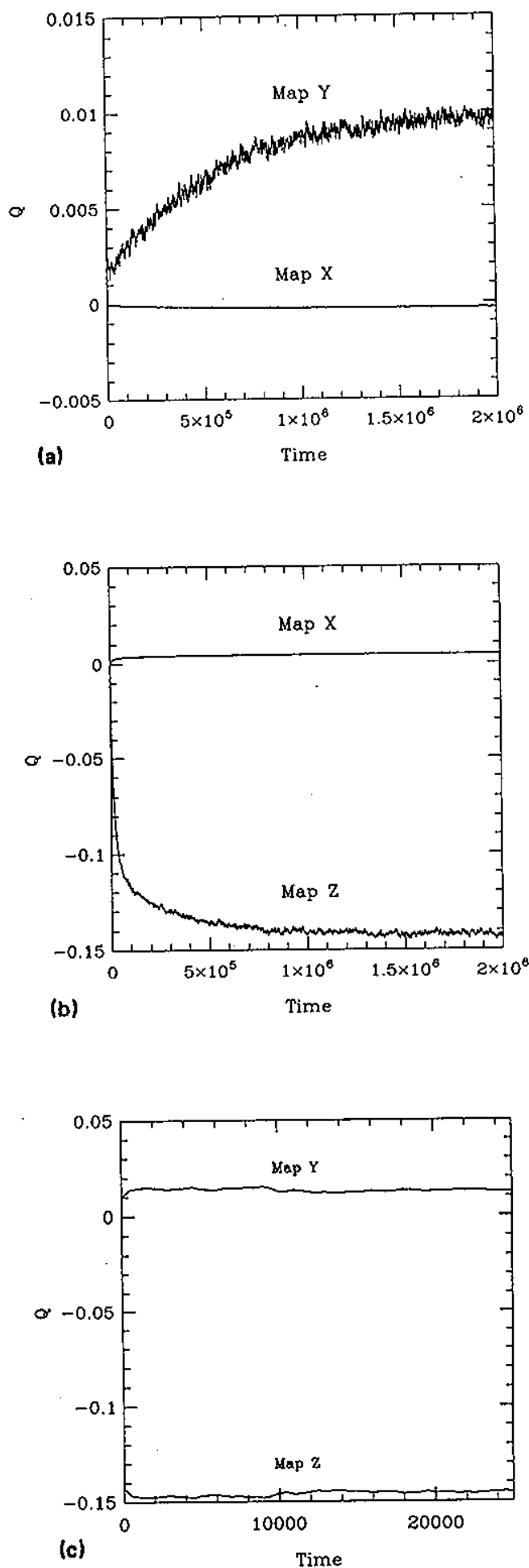

FIG. 7. Time evolution of means for model A for three maps $X, Y$, and $Z$, where two maps, in turn, are coupled linearly with strength $d=0.02$. All maps have $a=0.4: X$ has $L=4096$ and $b=1.3 ; Y$ has $L=128$ and $b=1.25 ; Z$ has $L=128$ and $b=2.0$. (a) $Y$ coupled to $X$; both maps have $Q=0$ initially. (b) $Z$ coupled to $X$; both maps have $Q=0$ initially. (c) $Y$ coupled to $Z$; the initial means are the asymptotic means generated from the coupling to $X$.

approximately as $\left(1 / L_{x}\right)^{1 / 2}$ (cf. Fig. 11). Within the error bars, the extrapolated means agree with those obtained from the heat-bath experiments described previously.

While the numerical experiments described above 


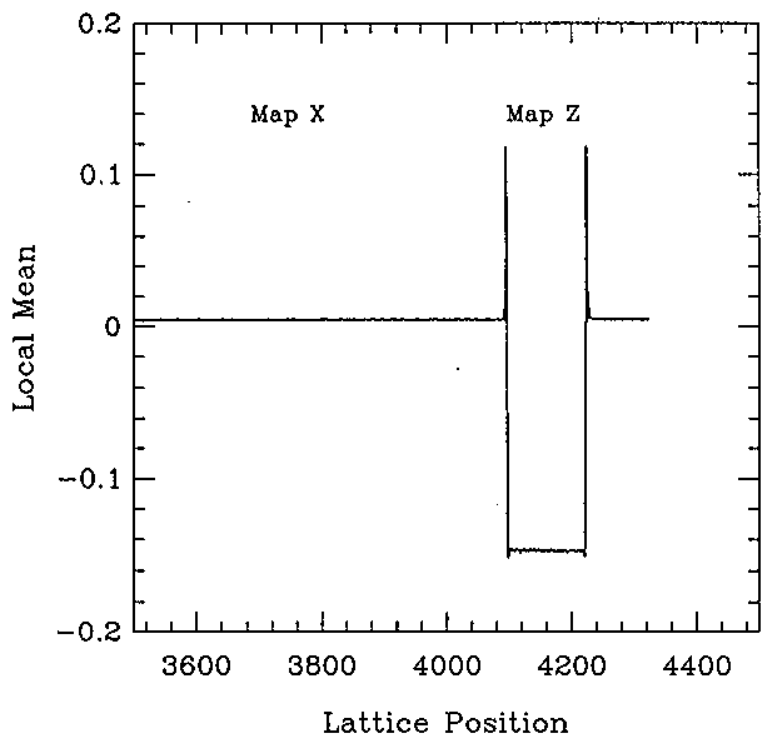

FIG. 8. Time average of local mean as a function of lattice position $i$, for two maps $X$ and $Z$. The lattice parameters are as described in Fig. $7(\mathrm{~b})$.

strongly suggest that thermodynamic considerations may be applicable to the coupled map models considered here, the straightforward application of "standard" thermodynamic ideas is difficult. As an example of these difficulties, we attempt to apply the Einstein fluctuation theorem: ${ }^{17}$ If there exists an entropy function $S$ which depends only on the conserved quantity $Q$, then small fluctuations of $Q$ about its equilibrium value should have a Gaussian distribution of width $\partial^{2} S / \partial Q^{2}$. Measurement of the fluctuations as a function of $Q$ should then provide an indirect way to measure the entropy. Now, if $S$ and $Q$ are extensive quantities, it follows that the mean-square fluctuations should scale as $1 / L$. However, for model $\mathrm{A}$ we find that the fluc-

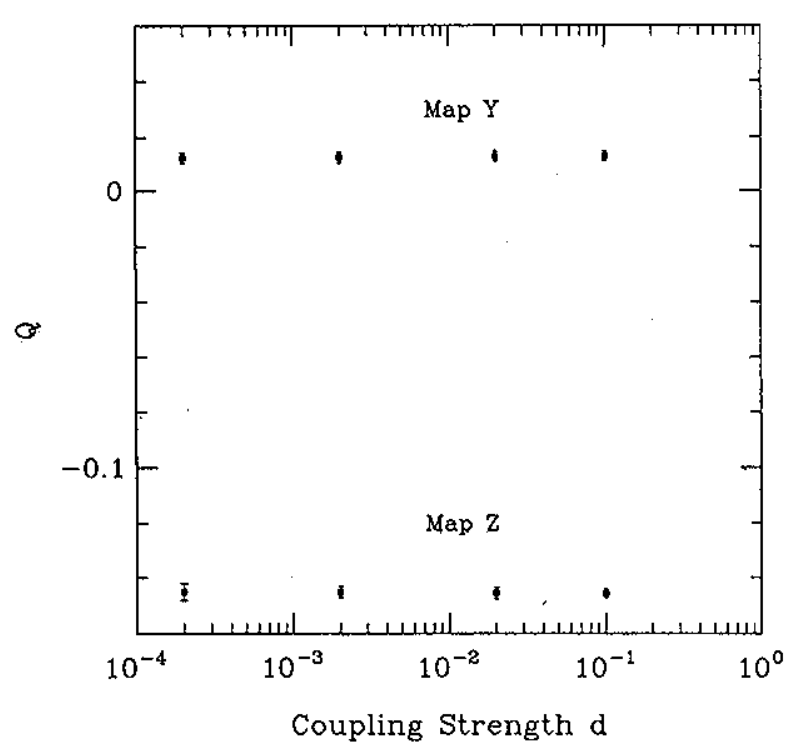

FIG. 9. Here, $Q$ in $Y$ and $Z$ when equilibrium has been achieved, as a function of coupling strength $d$. Lattice parameters are as in Fig. 7(c).

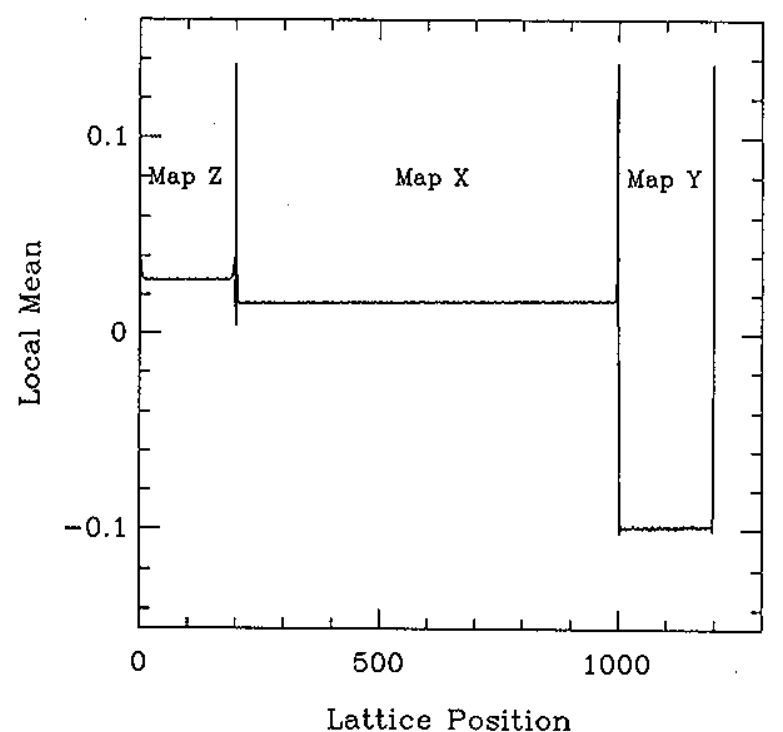

FIG. 10. Time average of local mean in model $A$ as a function of lattice position $i$, for three maps $X, Y$, and $Z$. The lattice parameters are: $L_{y}$ $=L_{z}=200, L_{x}=800 ; a_{x}=a_{y}=a_{z}=0.4 ; b_{x}=1.4, b_{y}=1.3, b_{z}=2.0$. The coupling between lattices is linear with strength $d=0.02$.

tuations instead scale as $1 / L^{2}$ (cf. Fig. 12). This result can be traced to the $k^{2}$ behavior of the structure factor $S_{k}$ at long wavelengths [cf. Fig. 4(b)]. To show this, consider the mean-square value $\sigma_{N}$ of the coarse-grained lattice amplitude fluctuations, averaged over $N$ sites, where $1 \ll N \ll L$ :

$$
\sigma_{N}=\left\langle\left(\frac{1}{N} \sum_{j=1}^{N} y_{j}\right)^{2}\right\}
$$

where, for simplicity, we assume the fluctuations are about zero mean. By simple manipulations, $\sigma_{N}$ can be related to the structure factor $S_{k}$ :

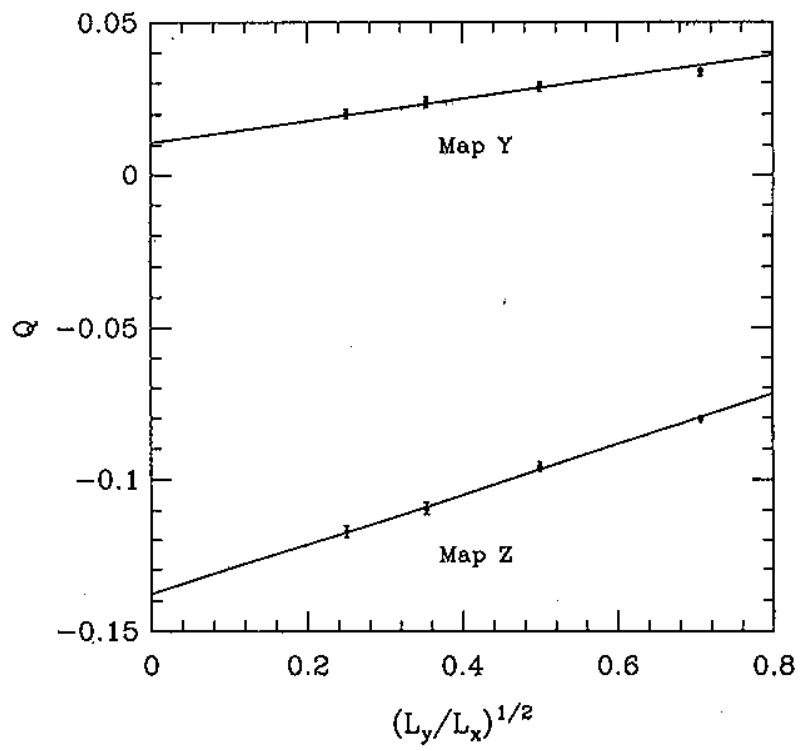

FIG. 11. Dependence of $Q_{y}$ and $Q_{2}$ for the maps described in Fig. 10, as a function of $L_{x}$. 




FIG. 12. Mean-square fluctuations of a map $X$ with $a_{x}=0.4$ and $b_{x}$ $=1.28$, coupled linearly to a map $Y$ with $a_{y}=0.4$ and $b_{y}=1.5$, as a function of the lattice size $L_{x}=L_{y}$.

$$
\sigma_{N}=\frac{1}{N^{2}} \frac{1}{L} \sum_{k=0}^{1 / 2} \frac{\sin ^{2}(\pi N k)}{\sin ^{2}(\pi k)} S_{k}
$$

For model A, $S_{k} \sim \sin ^{2}(\pi k)$ [cf. Fig. 4(b)], and therefore $\sigma_{N} \sim 1 / N^{2}$. If an entropy function indeed exists, the simple Taylor series approach underlying the Einstein fluctuation theorem appears therefore not to be directly applicable.

\section{CONCLUSION}

We have investigated coupled map models with conservation laws as possible model systems for chaos in ex. tended systems. Simple Langevin equations were found to adequately describe many of the long wavelength features of the models. We have resolved some important questions which had been raised ${ }^{15}$ concerning the applicability of the fluctuation-dissipation theorem to nonequilibrium systems. Finally, we have presented evidence that simple ideas from thermodynamics may be applicable to models without an underlying Hamiltonian.

Several questions raised in this work still remain to be resolved. The most immediate one is whether persistent currents seen in coupled map models may invalidate any analogies with thermodynamic equilibrium systems. More stringent tests of the Langevin equation for different symmetries and dimensionalities are also required. Further investigations are needed to clarify the applicability of entropy and dimension defined from a dynamical systems point of view, e.g., whether the Kaplan-Yorke conjecture relating dimension and Lyapunov exponents can be modified to be useful in situations where the sum of the Lyapunov exponents is positive.

\section{ACKNOWLEDGMENT}

This work was partially supported through NSF Grant No. DMR-9013984.

${ }^{1}$ G. Ahlers and R. P. Behringer, Prog. Theor. Phys. (Kyoto) Suppl. 64, 186 (1978); J. P. Gollub, A. R. McCarrior, and J. F. Steinmann, J. Fluid Mech. 125, 259 (1982); S. Ciliberto, J. Phys. Cond. Matter 2, SA483 (1990).

${ }^{2}$ N. B. Tufillaro, R. Ramshankar, and J. P. Gollub, Phys. Rev. Lett. 62, 422 (1989).

${ }^{3}$ For a review of maps on the unit interval, see P. Collet and J. P. Eckmann, Iterated Maps of the Interval as Dynamical Systems (Birkhäuser, Boston, 1980).

${ }^{4} \mathrm{~K}$. Kaneko, Physica D 37, 60 (1989), and references therein.

${ }^{5}$ Y. Kuramoto and T. Tsuzuki, Prog. Theor. Phys. 55, 356 (1976); G. I. Sivashinsky, Acta Astronaut. 6, 569 (1979).

${ }^{6}$ S. Zaleski, Physica D 34, 427 (1989).

${ }^{7}$ I. Shimada and T. Nagashima, Prog. Theor. Phys. 61, 1605 (1979), and references therein.

${ }^{8}$ J. L. Kaplan and J. A. Yorke, "Chaotic behavior of multidimensional difference equations," in Functional Differential Equations and Approximation of Fixed Points, edited by $\mathrm{H}$. O. Peitgen and $\mathrm{H}$. O. Walther, Lecture Notes in Mathematics, Vol. 730 (Springer-Verlag, New York, 1979).

${ }^{9} \mathrm{P}$. Manneville, "Lyapunov exponents for the Kuramoto-Sivashinsky model," in Macroscopic Modeling of Turbulent Flows, edited by $O$. Pironneau, Lecture Notes in Physics, Vol. 230 (Springer-Verlag, New York, 1985).

${ }^{10}$ D. Ruelle, Commun. Math. Phys. 87, 287 (1982). We thank G. Grinstein for calling this reference to our attention.

${ }^{11}$ For an introduction, see N. G. Van Kampen, Stochastic Processes in Physics and Chemistry (North-Holland, Amsterdam, 1981).

${ }^{12}$ Some results for 2-D conserving coupled maps are described in G. Grinstein, C. Jayaprakash, and B. Bolker, Phys. Rev. A 44, 4923 (1991). For related work on the Burgers equation, see, e.g., V. Yakhot and Z. S. She, Phys. Rev. Lett. 60, 1840 (1988); E. Medina, T. Hwa, M. Kardar, and Y. Zhang, Phys. Rev. A 39, 3053 (1989).

${ }^{13}$ P. C. Hohenberg and B. I. Halperin, Rev. Mod. Phys. 49, 435 (1977), and references therein.

${ }^{14}$ T. Hwa and M. Kardar, Phys. Rev. Lett. 62, 1813 (1989).

${ }^{15}$ P. C. Hohenberg and B. I. Shraiman, Physica D 37, 109 (1989).

${ }^{16}$ See, for instance, D. Forster, Hydrodynamic Fluctuations, Broken Symmetry and Correlation Functions (Benjamin, New York, 1975).

${ }^{17}$ See, e.g., L. Landau and E. M. Lifshitz, Statistical Physics (Pergamon, New York, 1980), 3rd ed., Part 1. 